This is an electronic reprint of the original article. This reprint may differ from the original in pagination and typographic detail.

\author{
Author(s): Carroll, R.J.; Page, R.D.; Joss, D.T.; Uusitalo, Juha; Darby, I.G.; Andgren, K.; Cederwall, \\ B.; Eeckhaudt, Sarah; Grahn, Tuomas; Gray-Jones, C.; Greenlees, Paul; Hadinia, B.; \\ Jones, Peter; Julin, Rauno; Juutinen, Sakari; Leino, Matti; Leppänen, Ari-Pekka; Nyman, \\ Markus; O’Donnell, D.; Pakarinen, Janne; Rahkila, Panu; Sandzelius, Mikael; Sarén, Jan; \\ Scholey, Catherine; Seweryniak, D.; Simpson, J.
}

Title: $\quad$ Competing Decay Modes of a High-spin Isomer in the Proton-unbound Nucleus 158Ta

Year: $\quad 2015$

Version:

Please cite the original version:

Carroll, R.J., Page, R.D., Joss, D.T., Uusitalo, J., Darby, I.G., Andgren, K., Cederwall, B., Eeckhaudt, S., Grahn, T., Gray-Jones, C., Greenlees, P., Hadinia, B., Jones, P., Julin, R., Juutinen, S., Leino, M., Leppänen, A.-P., Nyman, M., O’Donnell, D., . . Simpson, J. (2015). Competing Decay Modes of a High-spin Isomer in the Proton-unbound Nucleus 158Ta. Acta Physica Polonica B, 46(3), 695-698.

https://doi.org/10.5506/APhysPolB.46.695

All material supplied via JYX is protected by copyright and other intellectual property rights, and duplication or sale of all or part of any of the repository collections is not permitted, except that material may be duplicated by you for your research use or educational purposes in electronic or print form. You must obtain permission for any other use. Electronic or print copies may not be offered, whether for sale or otherwise to anyone who is not an authorised user. 


\title{
COMPETING DECAY MODES OF A HIGH-SPIN ISOMER IN THE PROTON-UNBOUND NUCLEUS ${ }^{158} \mathrm{Ta}^{*}$
}

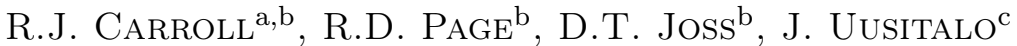 \\ I.G. Darby ${ }^{b}$, K. AndGren ${ }^{d}$, B. Cenderwall ${ }^{d}$, S. Eeckhaudt ${ }^{c}$ \\ T. Grahn ${ }^{\mathrm{c}}$, C. Gray-Jones ${ }^{\mathrm{b}}$, P.T. Greenlees ${ }^{\mathrm{c}}$, B. Hadinia $^{\mathrm{d}}$

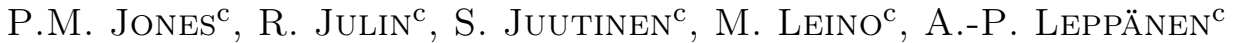

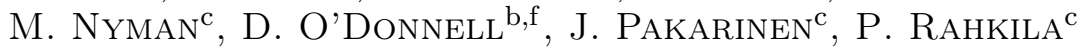 \\ M. Sandzelius ${ }^{\mathrm{c}}$, J. Sarén ${ }^{\mathrm{c}}$, C. Scholey ${ }^{\mathrm{c}}$, D. SeweryniaK ${ }^{\mathrm{e}}$ \\ J. SIMPSON ${ }^{\mathrm{f}}$ \\ ${ }^{a}$ Department of Physics, University of Surrey, Guildford, GU2 7XH, UK \\ ${ }^{\mathrm{b}}$ Department of Physics, Oliver Lodge Laboratory, University of Liverpool \\ Liverpool, L69 7ZE, UK \\ ${ }^{\mathrm{c}}$ Department of Physics, University of Jyväskylä \\ P.O. Box 35, 40014, Jyväskylä, Finland \\ ${ }^{\mathrm{d}}$ Department of Physics, Royal Institute of Technology, 10691 Stockholm, Sweden \\ eArgonne National Laboratory, Physics Division, Argonne, Illinois 60439, USA

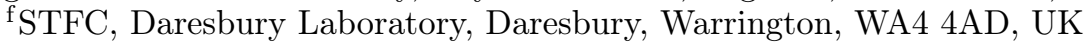

(Received January 29, 2015)

An isomeric state at high spin and excitation energy was recently observed in the proton-unbound nucleus ${ }^{158} \mathrm{Ta}$. This state was observed to decay by both $\alpha$ and $\gamma$ decay modes. The large spin change required to decay via $\gamma$-ray emission incurs a lifetime long enough for $\alpha$ decay to compete. The $\alpha$ decay has an energy of $8644(11) \mathrm{keV}$, which is among the highest observed in the region, a partial half-life of $440(70) \mu$ s and changes the spin by $11 \hbar$. In this paper, additional evidence supporting the assignment of this $\alpha$ decay to the high-spin isomer in ${ }^{158} \mathrm{Ta}$ will be presented.

DOI:10.5506/APhysPolB.46.695

PACS numbers: 23.20.Lv, 23.35.+g, 23.50.+z, 23.60.+e

\section{Introduction}

The recent observation of an isomer at high spin, $19^{-}$, and excitation energy, $2809 \mathrm{keV}$, in the proton-unbound nucleus ${ }^{158} \mathrm{Ta}$ [1] raised the possibility of a blurring to the limits of the observable nuclear landscape due to the possible existence of isomers. These isomers can be sufficiently long

\footnotetext{
* Presented at the Zakopane Conference on Nuclear Physics "Extremes of the Nuclear
} Landscape", Zakopane, Poland, August 31-September 7, 2014. 
to survive a separator flight time and hence be observed at the focal plane. Both $\alpha$ - and $\gamma$-decay modes have been associated with this isomer, as shown in Fig. 1. In this paper, additional experimental evidence supporting the previous assignment of a new $\alpha$ decay to this isomer will be presented.

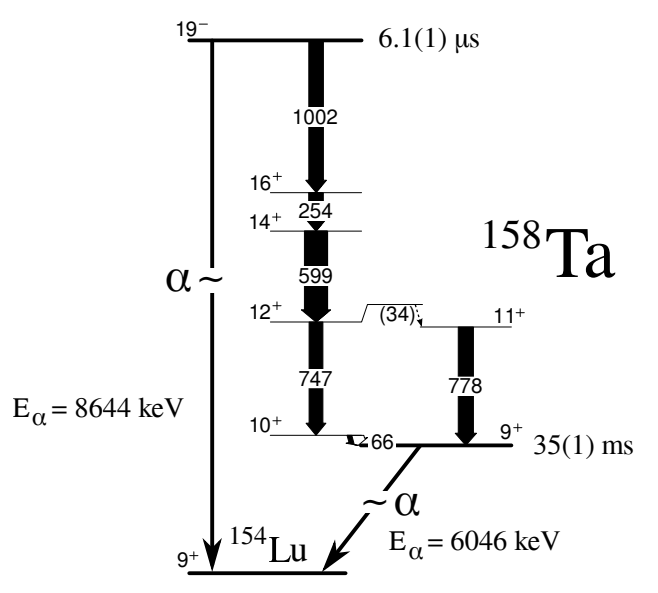

Fig. 1. Partial level scheme of ${ }^{158} \mathrm{Ta}$ including competing decay branches from the $19^{-}$isomer. Both $\alpha$ - and $\gamma$-decay branches lead to the population of ${ }^{154} \mathrm{Lu}$. Transition energies are in $\mathrm{keV}$.

\section{Experimental details}

The experiment was performed at the University of Jyväskylä accelerator laboratory. The ${ }^{158} \mathrm{Ta}$ nuclei were produced in excited states using fusionevaporation reactions induced by ${ }^{58} \mathrm{Ni}$ ions, with a beam energy of $255 \mathrm{MeV}$, incident on an isotopically enriched ${ }^{102} \mathrm{Pd}$ target of thickness $\sim 1 \mathrm{mg} \mathrm{cm}^{-2}$. The JUROGAM HPGe spectrometer surrounded the target position and was used to measure prompt $\gamma$-ray emissions. The RITU gas-filled separator [2] transported recoiling reaction products to its focal plane and also suppressed unreacted beam. The GREAT spectrometer [3] was situated at the focal plane. Recoiling nuclei that entered GREAT passed through a multiwire proportional counter (MWPC) before being implanted into one of two adjacently mounted double-sided silicon strip detectors (DSSDs). Subsequent radioactive $\alpha$ decays were detected by the DSSDs but not the MWPC, thus distinguishing between signals associated with recoils and decays. A planar and a Clover Ge detector were used to measure X-rays and $\gamma$-rays from the DSSDs that were emitted during decay processes. Data were recorded using a triggerless data acquisition system [4], time stamped with a precision of $10 \mathrm{~ns}$, and events were built in software [5]. Reaction channels were identified using standard tagging techniques $[6,7]$. 


\section{Evidence for the $\alpha$-decay branch}

Gamma-ray transitions observed at the focal plane revealed the presence of the isomer at high spin and excitation energy, which primarily $\gamma$ decays via a $1002 \mathrm{keV}$ transition [1]. A new $\alpha$ decay $\left(\mathrm{E}_{\alpha}=8644(11) \mathrm{keV}\right)$ was observed to decay with a half-life similar to that of this isomer. The decay curves of the $\alpha$ - and $\gamma$-decay branches are compared in Fig. 2 (a)-(b). The measured half-life of the $\alpha$-decay branch is 6.4(4) $\mu \mathrm{s}$, which is consistent with the 6.1(1) $\mu$ s half-life associated with the $\gamma$-decay branches. The same $\gamma$-ray transitions feeding the isomer are observed in association with both the $\alpha$ - and $\gamma$-decay branches. Based on this evidence, the new $\alpha$ decay was assigned to the same high-spin isomer.
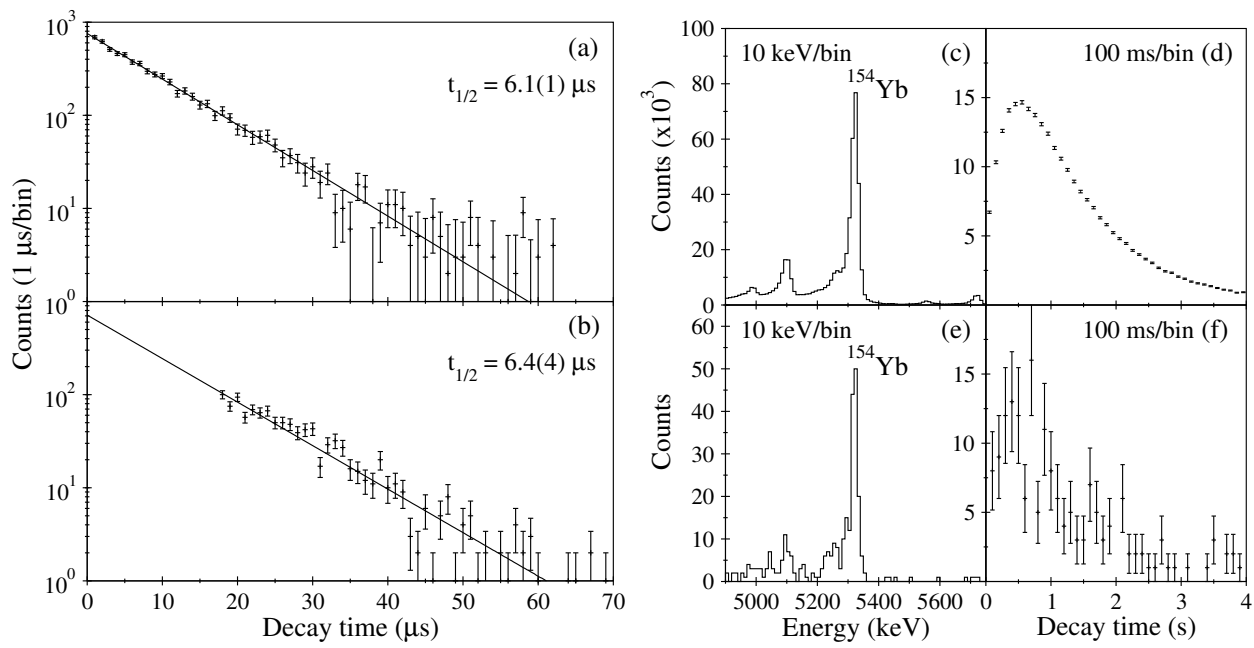

Fig. 2. Decay curves for (a) the $1002 \mathrm{keV} \gamma$-ray transition and (b) the $8644 \mathrm{keV}$ $\alpha$ decay, which have consistent half-lives. (c) The energy and (d) decay time of decays following the $\alpha$ decay of the $9^{+}$state in ${ }^{158} \mathrm{Ta}$. (e) The energy and (f) decay time of decays following the $8644 \mathrm{keV} \alpha$ decay from the high-spin isomer in ${ }^{158} \mathrm{Ta}$. The $5331 \mathrm{keV}{ }^{154} \mathrm{Yb}$ peak appears strongly above the background in both (c) and (e). The ${ }^{154} \mathrm{Yb}$ decay times in (d) and (f) reveal the unobserved $\beta$ decay of ${ }^{154} \mathrm{Lu}$, completing the decay chain from ${ }^{158} \mathrm{Ta} \rightarrow{ }^{154} \mathrm{Lu} \rightarrow{ }^{154} \mathrm{Yb} \rightarrow{ }^{150} \mathrm{Er}$, and have consistent peaks. These similarities reinforce the assignment of the $8644 \mathrm{keV}$ $\alpha$ decay to the $19^{-}$isomer in ${ }^{158} \mathrm{Ta}$.

Further evidence that this $\alpha$ decay originates from ${ }^{158} \mathrm{Ta}$ can seen in the subsequent decays, which are shown in Fig. 2 (c)-(f). The $\gamma$-decay branches of the isomer feed the $9^{+}$low-lying metastable state. The decay of this state is the first step in the following decay chain:

$$
{ }^{158} \mathrm{Ta}_{9^{+}} \rightarrow \alpha(6046) \rightarrow{ }^{154} \mathrm{Lu}_{9^{+}} \rightarrow \beta^{+} \rightarrow{ }^{154} \mathrm{Yb}_{0^{+}} \rightarrow \alpha(5331) \rightarrow{ }^{150} \mathrm{Er}_{0^{+}},
$$


of which, in this experiment, only the $\alpha$ decays could be observed. The $5331 \mathrm{keV}{ }^{154} \mathrm{Yb} \alpha$ decay [8] is observed strongly above the background following the decay of the $9^{+}$state in ${ }^{158} \mathrm{Ta}$. Furthermore, the decay curve reveals the unobserved $\beta$-decay component from ${ }^{154} \mathrm{Lu}$. A similar energy and decay curve can be seen following the decay of the $8644 \mathrm{keV} \alpha$ decay, which suggests that it feeds the same decay chain, and thus originates from ${ }^{158} \mathrm{Ta}$. A closed $Q$-value loop incorporating the $\alpha$ - and $\gamma$-decay branches depopulating the ${ }^{158} \mathrm{Ta}_{19^{-}}$isomer and populating the ${ }^{154} \mathrm{Lu}_{9+}$ state is evidence that the $8644 \mathrm{keV} \alpha$ decay is a direct transition between these two states [1]. The total $Q$-values via the $\alpha$-decay branch and via the $\gamma$-ray branch are $8869(11)$ and $8870(14)$, respectively. To account for the change in spin and parity, an angular momentum change of $11 \hbar$ occurs as a result of this decay.

\section{Summary and acknowledgements}

The $8644 \mathrm{keV} \alpha$ decay was previously assigned to the $19^{-}$isomer in ${ }^{158} \mathrm{Ta}$ based on the feeding $\gamma$-ray transitions, the half-life and the $Q$-value, all of which are consistent with observations associated with the $\gamma$-decay branch. The subsequent radioactive decay data presented in this paper is consistent with a decay from ${ }^{158} \mathrm{Ta}$, which reinforces the previous assignment.

This work has been supported through the UK Science and Technology Facilities Council, the Academy of Finland under the Finnish Centre of Excellence Programme 2006-2011 (Nuclear and Accelerator Based Physics contract 213503), EURONS (European Commission contract No. RII3-CT2004-506065) and the U.S. Department of Energy, Office of Nuclear Physics, under contract No. DEAC02-06CH11357. The UK/France (STFC/IN2P3) Loan Pool and GAMMAPOOL network are acknowledged for the EUROGAM detectors of JUROGAM. T.G., P.T.G. and C.S. acknowledge the support of the Academy of Finland, contract numbers 131665, 111965 and 209430, respectively.

\section{REFERENCES}

[1] R.J. Carroll et al., Phys. Rev. Lett. 112, 092501 (2014).

[2] M. Leino et al., Nucl. Instrum. Methods Phys. Res. B99, 653 (1995).

[3] R.D. Page et al., Nucl. Instrum. Methods Phys. Res. B204, 634 (2003).

[4] I.H. Lazarus et al., IEEE Trans. Nucl. Sci. 48, 567 (2001).

[5] P. Rahkila, Nucl. Instum. Methods Phys. Res. A595, 637 (2008).

[6] K.-H. Schmidt et al., Phys. Lett. B168, 39 (1986).

[7] E.S. Paul et al., Phys. Rev. C51, 78 (1995).

[8] R.D. Page et al., Phys. Rev. C53, 660 (1996). 